\title{
Leap Motion-Enhanced Augmented Reality Video Games to Encourage Intergenerational Engagement and Wrist Exercise
}

\author{
Hong-Wei Wong, Chien-Sing Lee \\ Sunway University, Malaysia, hong.w2@imail.sunway.edu.my
}

\begin{abstract}
For years, the health industry has been urging people to live a healthy life either by exercising or eating healthily. Furthermore, often, retired seniors would like to play with their family but there is not much common interest. In addition, one of the acute health problems at the workplace is carpal tunnel syndrome. Hence, inspired by Kinect and Wii, this project aims to: a) encourage more intergenerational family-senior engagement and $b$ ) encourage exercise at the workplace whenever they need a break. A video game gives a sense of achievement and progress is visible. Thus, we have developed Leap-Motion-enhanced games such as rolling ball, trivia, and maze video games of different levels to cater to different abilities. We also hope that these games would add to the activities in retirement homes/villages. The theme and objects in the games can be easily changed. Thus, the system can be easily personalized for different socio-cognitive-affective engagement. Feedback from the Leap Motion-enhanced developer community is positive and constructive with areas for improvement.
\end{abstract}

Key words: Augmented Reality Video Games, Intergenerational Engagement, Leap Motion, Wrist Exercise.

\section{INTRODUCTION}

Physical in activity increases for senior citizens, due to greater in-mobility as aging progresses and sometimes, due to lack of motivation to exercise. Furthermore, Macera, Hootman and Sniezek [1] note that to live a painless and easy life at old age can sometimes be costly. There are also many associated psychological effects such as depression, and loneliness, sometimes due to the empty nest syndrome.

Scanlon-Mogel and Roberto [2] find that senior citizens mainly want to age well and to be treated in the same way as when they were younger. It is thus important to introduce affordable means to exercise which also encourages social-cognitive-affective engagement.
Another aspect we are interested in is wrist exercise. This is because wrist exercise can be helpful to seniors suffering from Rheumatoid Arthritis (RA) and working adults suffering from Carpal Tunnel Syndrome.

Cooney, Law, Matschke, Lemmey, Moore, Ahmad, Jones, Maddison and Thom [3] highlight that Rheumatoid arthritis (RA) is the most common form of chronic joint inflammation that affects individuals as young as 40 years old. The risk is five times higher in women. The effects of RA are severe joint pain and reduced muscle strength. Hammond and Prior [4] recommend hand exercises at home as part of RA management.

Carpal Tunnel Syndrome (CTS) on the other hand is a syndrome, which involves compression of the median nerve in the carpal tunnel of the wrist. The effects are numbness and discomfort of muscles surrounding the wrist area of the hand. To relieve the numbness caused by Carpal Tunne 1Syndrome,Muller, Tsui, Schnurr, Biddulph-Deisroth, Hard, and MacDermid's [5] research in Ontario surveyed various types of therapy ranging from physical to medication. Findings reveal that out of these, physical therapy including yoga and most importantly nerve gliding exercises, are the most effective.

Leap Motion is well-suited to creating interesting experiences to encourage cognitive and physical (wrist)exercises. It allows interaction not only with 2Dbutalso3Dinterfaces. For instance, the device could capture the user's every finger, joint and every hand posture as pointed out by Plemmon and Mandel [6]. Hence, it could be included into the category of Augmented, Virtual and Mixed Reality.

Furthermore, being a natural user interface (NUI) device, Leap Motion enables more natural control compared to conventional game controllers. Thus, interactions are learnable habits whereby once familiar, the user can control without thinking.

\subsection{Objectives}

The objectives of this research paper are to increase senior citizens' and workplace adults' cognitive-affective-physical 
activity through Leap Motion-enhanced video games. Social engagement with family members can be developed through future development of these Leap Motion games to multi-player competitive games.

\section{RELATED WORK}

Prevention is better than cure. Thus, for years, the health industry has been urging people to live a healthy life either by exercising or eating healthily. As health issues mostly occur due to many years of unhealthy lifestyle, exercising even a little daily could help to make a difference. Our focus is on developing video games for seniors and working adults that promotes problem-solving and wrist exercises (in the current version), and in the future, social engagement (multiplayer mode).

In the movies, people dream of having the ability to interact intuitively just like Tony Stark in the Iron Man movies. Such controls are human-like and most importantly it feels real to the user. With computers, many peripherals are created/developed to obtain the desired input and control. Popular motion control devices in the game industry are Microsoft Kinect and Nintendo Wii. Inspired by Kinect and Wii, this project encourages more exercise through Leap Motion.

Another motivating element is gaming and gamification. A game gives a sense of achievement which people can obtain and is visible to them. People tend to be demotivated if they cannot see progress or if they cannot achieve their goals. Hence, the progress/goal indicators are important to sustain interest.

Incorporation of gamification mechanics in the developed game would motivate users further. Lister, West, Cannon, Sax and Brodegard's [7] study investigates the extent to which gamification is used in health apps, and the potential of gamification in health and fitness apps in influencing consumer's health behaviour. Based on 132 health diet and fitness apps in the Apple App Store in the winter of 2014, 10 effective game elements, the 6 core components of health gamification, and 13 core health behaviour constructs are analysed using regression analysis.

Findings indicate that game elements are associated with gamification $(p<.001)$, behavioural theory is associated with gamification $(p<.05)$, but not game elements, and gamification is associated with composite motivational behaviour scores $(p<.001)$, but not capacity or opportunity/trigger.

The association between gamification, usage of health applications and fitness highlights that the game mechanics of rewards and achievements are sufficient to sustain the interest of players besides high-quality graphics in the 3D environment and story-telling mechanisms. However, more important is the need to integrate important elements of behavioral theory from the app industry, which can potentially impact the efficacy of gamification apps to change behavior[8].

Furthermore, as age increases, eye sensitivity worsens. The game design should therefore, be more colorful and aesthetic in appearance. Moreover, the overall type face should be clear. In addition, the game genre would be based on easy problem-solving, such as trivia and maze. Trivia games are interesting. Our trivia game complements Sunway City's Snap and Win challenge.

\section{METHODOLOGY}

Our video game development is based on agile software development methodology. The agile methodology allows iterations which include incremental enhancement and other modifications during the design and development phase.

The video game development is created in Unity 3D and Leap Motion SDK. Leap Motion is the controller and the V2 skeletal tracking SDK the gesture capture/ tracking enabler. Other game engines such as Unreal Engine does not support Leap Motion-based development well. Moreover, Unity 3D allows exporting to different platforms with ease if future development requires. Hence, Unity 3D would allow better device platform development.

\section{THE SYSTEM}

\subsection{Use case}

The use case for the system is presented in Figure 1.

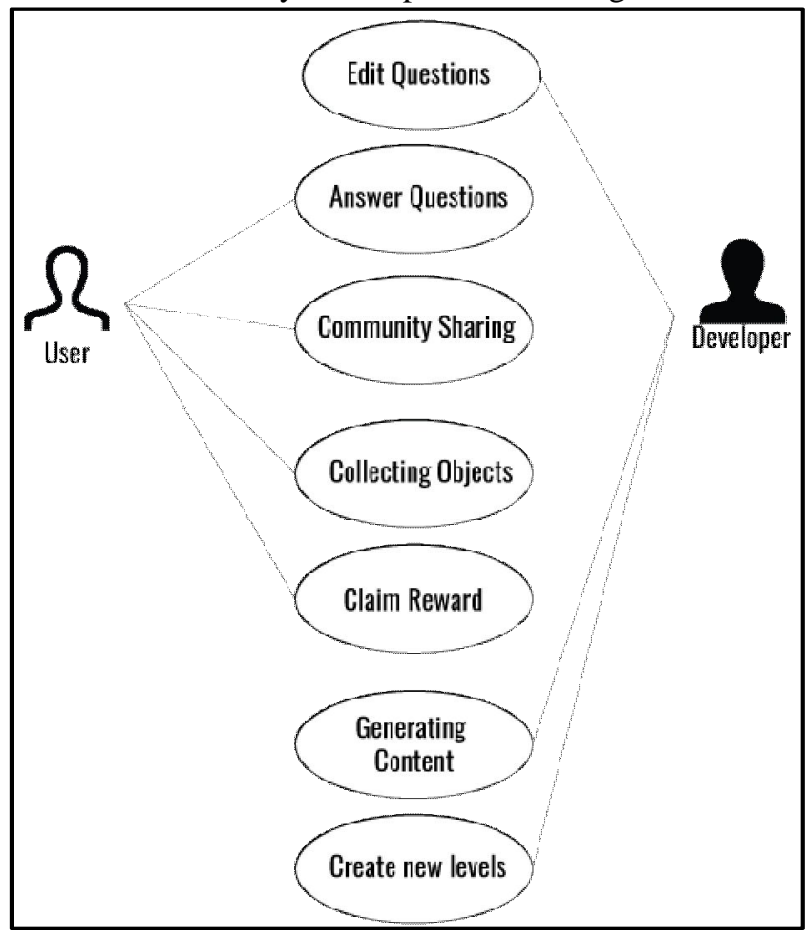

Figure 1: Use Case 


\subsection{Games}

The video game starts with the menu (Figure 2). Players can refer to the instructions by clicking on "How to Play". In the first two games (Figures 3 and 4), the main goal is to roll the ball via Leap Motion to collect a certain number of required objects within a time limit. The more objects collected, the more points. As the level increases, it gets more difficult and challenging.
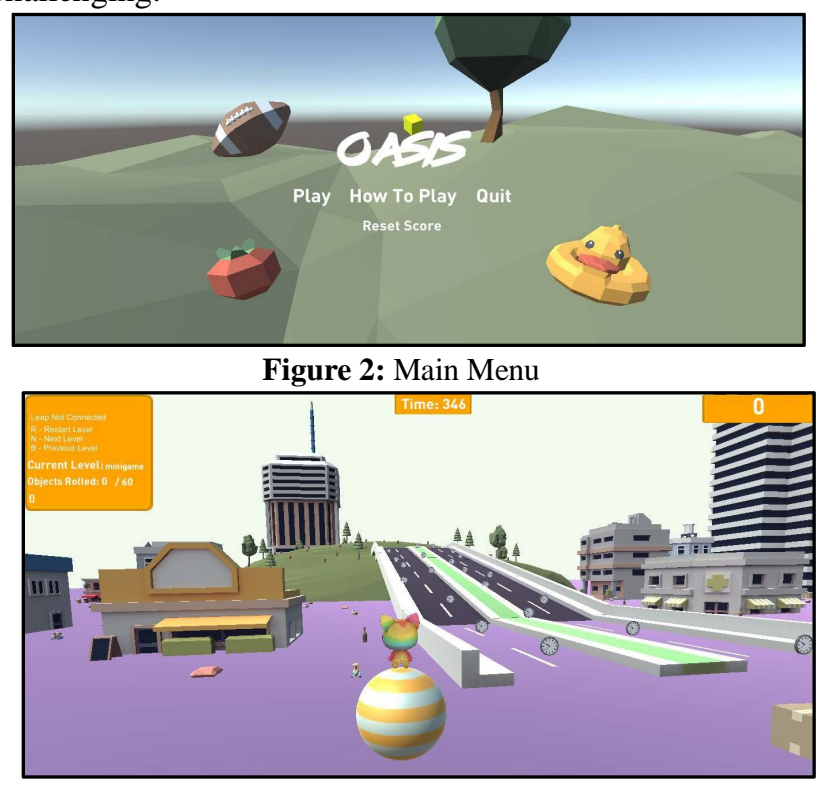

Figure 3: Controlling the Ball Based on Hand Direction via Leap Motion

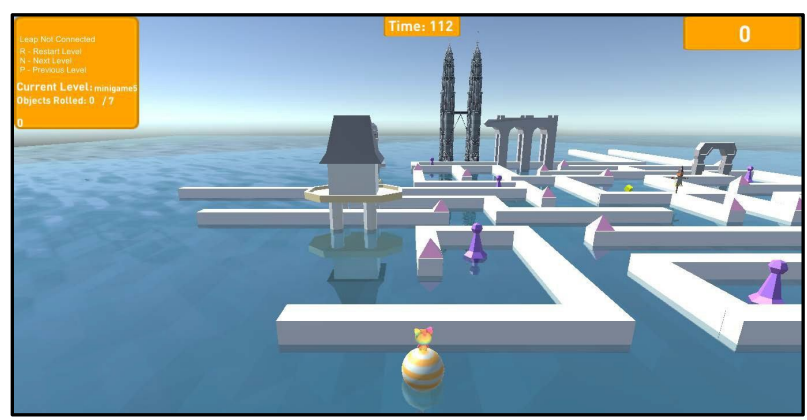

Figure 4: Rolling the Ball Through theMaze

For the trivia game (Figure 5), the player is required to grab the colored ball that matches the colored answer and place it into the empty barrel.

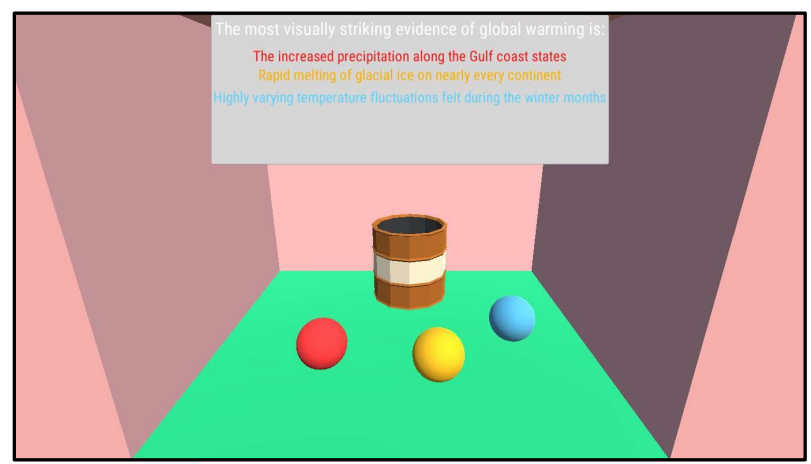

Figure 5: 3D Interaction with Virtual Objects with Leap Motion: Grab the Colored Ball and Place It in the Empty Barrel to Answer Questions
For each game, points are collected. If the player collects enough points, the player will be awarded with a real-life prize, i.e., a cash voucher, redeemable at the associated Website.

\section{RESULTS AND DISCUSSION}

\subsection{User testing}

\section{Sample: Senior Citizens}

We obtained two rounds of user feedback to gain insights from users. The first round of feedback was conducted at an open-air local carnival, specially held for senior citizens. There was low technology acceptance as most of the senior citizens were not tech-savvy. There were however, a few senior citizens who showed interest and tested out the video games.

The problem was mainly difficulty in controlling the Leap Motion device. The infrared sensor in Leap Motion performed poorly under bright light. The other factor was that the usage of Leap Motion required practice. The area of activation was not clear. Moreover, it was not easy to block the navigable area using a box. This may block out bright light but it would also create a mental "barrier" as the physical hand would be hidden and only the projected digital hand would be visible on the screen.

The senior citizens who played the video games however, showed interest in playing the games in the future when there are more improvements. One possible easy amendment is to provide a transparent/translucent "box" to better define the area that the hand can navigate.

\subsection{Feedback From The Leap Motion Developer Community}

Sample: Leap Motion developer community aged between 23-33.

This community was chosen as they are experts and would be able to provide constructive feedback such as on the user interface, content and new features. Their age distribution and profession are presented in Figures $6 a, b$. 
Hong-Wei Wong et al., International Journal of Advanced Trends in Computer Science and Engineering, 9(1.3), 2020,78 - 82
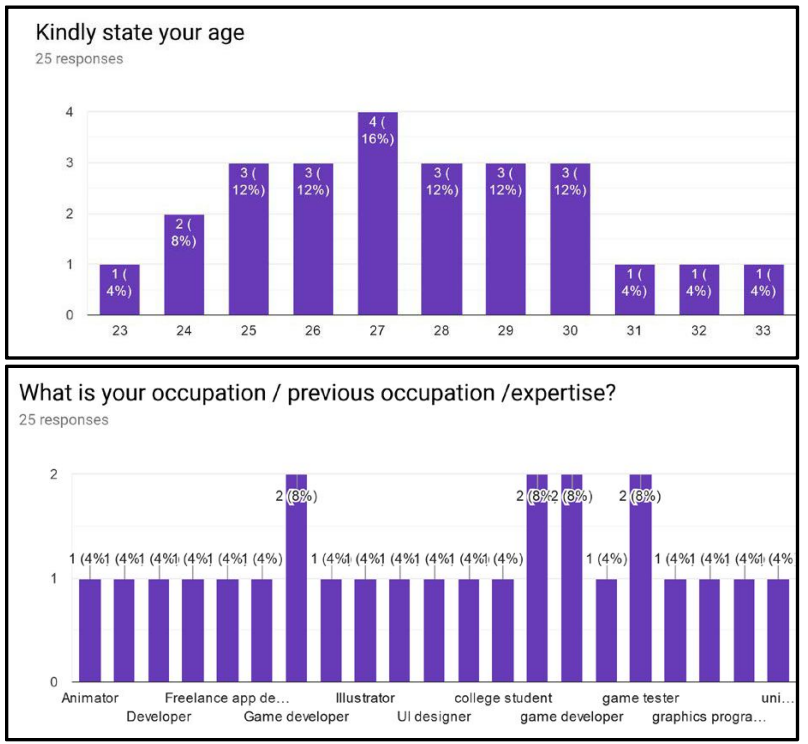

Figures 6a, b: Age and Profession

Findings indicate the following (Figures $7 \mathrm{a}-\mathrm{j})$ :

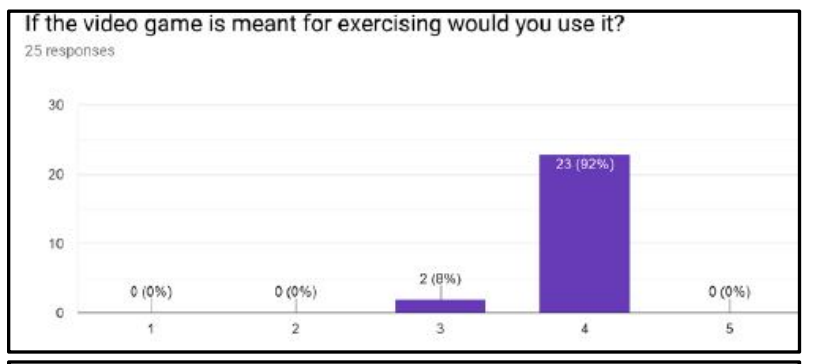

How attractive is the video game?

25 responses

30

20

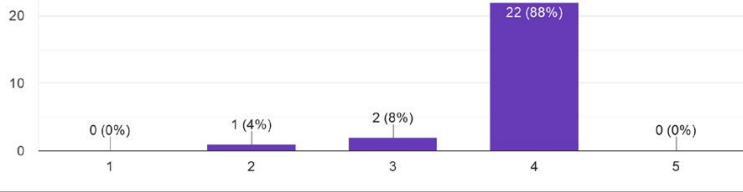

Do you find Leap Motion technology fun to use?

25 responses

30

20

20

10

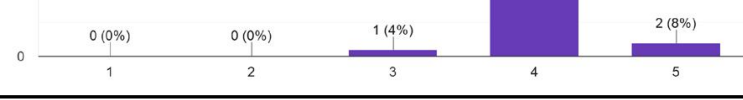

Is the video game understandable in usage?

25 responses

20

10

\begin{tabular}{ccc}
$0(0 \%)$ & $0(0 \%)$ & $1(4 \%)$ \\
\hline 1 & 2 & 3
\end{tabular}

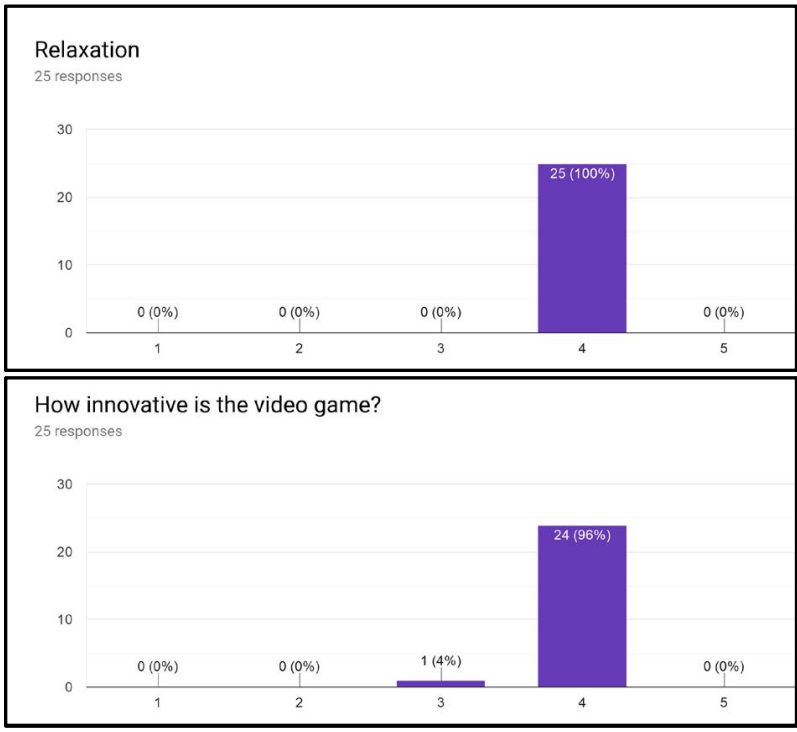

Do you find this video game usual compare to current available video game?

25 responses

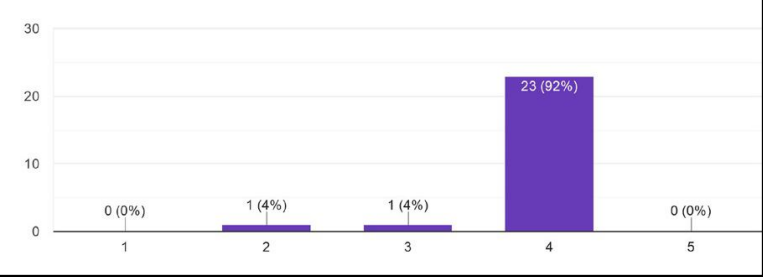

Did you gain useful information / knowledge from playing the video game? 25 responses
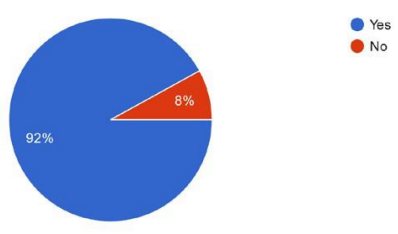

Do you find learning through video game interesting?

25 responses

30

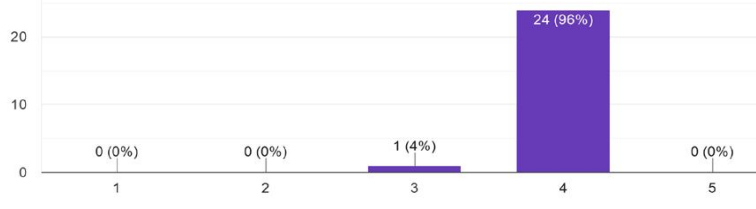

Will you be interested to play the video game in the future with further development?

25 responses

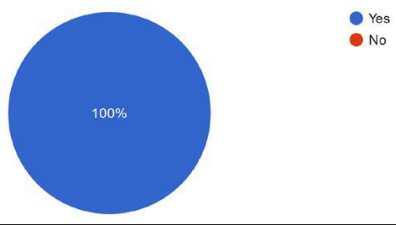

Figures 7a-j: Leap-Motion Developer Community Feedback 
Hong-Wei Wong et al., International Journal of Advanced Trends in Computer Science and Engineering, 9(1.3), 2020,78 - 82

Overall, findings are positive though with much room for improvement. Highlights are as follow:

- $76 \%$ of the respondents find the game is likeable. They like the overall design, gameplay and feel.

- $88 \%$ of the respondents find the game concept interesting

- $88 \%$ of the respondents find Leap Motion fun to use

- $96 \%$ of the respondents find the game is innovative in gameplay experience.

- $92 \%$ of the respondents find the game inventive

- $80 \%$ of the respondents find the game useful. Given improvements, all agreed that they would try out the game.

\section{CONCLUSION}

At the end of the day, one would ask does a development product and/or service work when introduced to the masses? Everything starts with an idea or a concept that later blooms into something usable. For this research, the main objective is to investigate whether our design integrating the idea of entertainment such as video game with the help of Leap Motion technology would help in behavior change such as increasing wrist exercise to alleviate RA and CTS.

Does our design work? The answer is both yes and no. To create a product that could fit all is not easy and is potentially impossible. As different age groups and generations see things differently, how products/ services are developed changes. In this case, the active senior citizens do not easily accept Leap Motion technology to make wrist exercise more interesting. Some cite disfamiliarity with the technology. Many prefer downloadable mobile apps as RA is not imminent yet. On the other hand, the younger aged group are more positive as they themselves are developers. Hence, video games can help in behavior change, if users find them useful.

Furthermore, the higher the "replayability" or replay value of a video game, the higher the acceptance. We have identified the games' strengths. These can be further improved on. In addition, just like learning, practice makes perfect. Moreover, based on the surveys, the challenge, competition, excitement and inventive aspects of the video games have to be improved to obtain better outcomes.

There are limitations to the study. The sample size for both groups are small. As such, the findings are preliminary. We need to conduct tests and surveys with a wider group of users after refining the system.

Thefutureofworkforthisresearchstudywouldmorelikelyinclu detheuseofupdatedsoftware in Leap Motion of Orion as it provides higher tracking accuracy. To motivate, the display of points/rewards and progress in a more attractive and visible manner complemented by sound would be included. Extending the system to a multiplayer game could also encourage more user interaction and community engagement.

\section{ACKNOWLEDGEMENT}

This research is partially supported by the Malaysian Fundamental Research Grant Scheme (FRGS) ref. FRGS/2016/ICT04/SYUC/01/1 with ethics approval SUREC 2016/067. The authors fully acknowledge the Ministry of (Higher) Education (MOHE) and Sunway University for the approved fund.

\section{REFERENCES}

1. Macera, C., Hootman, J. and Sniezek, J. 2003. Major public health benefits of physical activity. Arthritis \& Rheumatism, 49(1): 122-128. https://doi.org/10.1002/art.10907

2. Scanlon-Mogel, J. and Roberto, K. 2004. Older adults' beliefs about physical activity and exercise: Life course influences and transitions. Quality in Ageing and Older Adults, 5(3): 33-44.

3. Cooney, J., Law, R., Matschke, V., Lemmey, A., Moore, J., Ahmad, Y., Jones, J., Maddison, P. and Thom, J. 2011. Benefits of Exercise in Rheumatoid Arthritis. Journal of Aging Research, 1-14. https://doi.org/10.4061/2011/681640

4. Hammond, A. and Prior, Y. 2016. The effectiveness of home hand exercise programmes in rheumatoid arthritis: a systematic review. British Medical Bulletin, 119(1): 49-62. https://doi.org/10.1093/bmb/ldw024

5. Muller, M., Tsui, D., Schnurr, R., Biddulph-Deisroth, L., Hard, J. and MacDermid, J. 2004. Effectiveness of hand therapy interventions in primary management of carpal tunnel syndrome: a systematic review. Journal of Hand Therapy, 17(2): 210-228. https://doi.org/10.1197/j.jht.2004.02.009

6. Plemmons, D. and Mandel, P. 2016. Introduction to Motion Control. [online] Developerarchive.leapmotion.com. Available at: https://developer-archive.leapmotion.com/articles/intro-t o- motion-control Leap Motion. Technology.

7. Lister, C., West, J., Cannon, B., Sax, T. and Brodegard, D. 2014. Just a Fad? Gamification in Health and Fitness Apps. JMIR Serious Games, 2(2): p. e9. https://doi.org/10.2196/games.3413

8. C.Anitha, R. Mathusoothana S. Kumar. Extremely Low Light Video Enhancement along with No-Reference Video Quality Measurements. International Journal of Advanced Trends in Computer Science and Engineering. Volume 8, No.5, September - October 2019 https://doi.org/10.30534/ijatcse/2019/42852019 